\title{
Blocking the neurokinin 1 receptor reduces anxiety and pain in women with IBS
}

A proof of concept pilot study has shown that the areas of the brain associated with pain and anxiety have reduced activation following a painful rectal stimulus in patients treated with a neurokinin 1 receptor (NK1R) antagonist compared with control individuals given placebo.

IBS is a common disorder, but can be difficult to treat as the current pharmacological options are limited. "NK1R has been considered a potential target for treating IBS due to the involvement of this system in both sensitized pain and anxiety-common features of IBS," explains corresponding author Kirsten Tillisch. The researchers recruited 16 female patients with IBS, of whom 11 completed the study. The

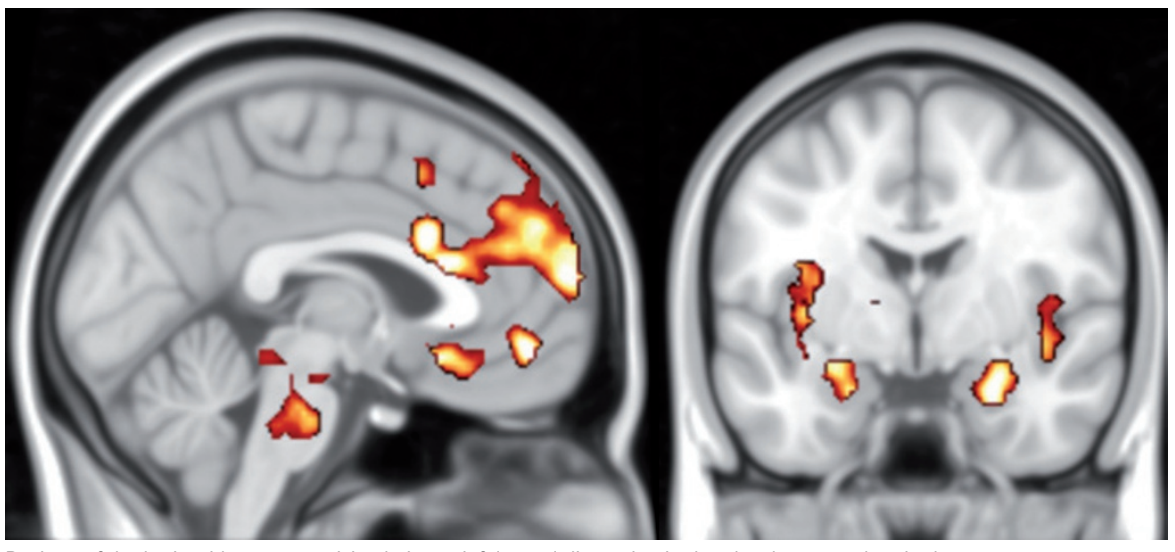

Regions of the brain with greater activity during painful rectal distension in the placebo group than in the treatment group, including the amygdala, hippocampus, anterior midcingulate gyrus and posterior insula. Courtesy of K. Tillisch. patients were treated with an NK1R antagonist, after which they self-reported their pain and anxiety and underwent a functional MRI session that included rectal balloon distension.

The drug decreased activity in areas of the brain that are associated with emotional arousal and in the interoceptive network. Patients who received the drug also reported lower anxiety levels and pain ratings than those who received placebo. Although the compound used in the study is no longer being developed, these findings suggest that antagonists of NK1R could be effective treatments of anxiety-related symptoms in some patients with IBS.

\section{Claire Greenhill}

Original article Tillisch, K. et al. Neurokinin-1-receptor antagonism decreases anxiety and emotional arousal circuit response to noxious visceral distension in women with irritable bowel syndrome. Aliment. Pharmacol. Ther. 35 , 360-367 (2012) 